\title{
Communicating Hydrocephalus Onset Following a Traumatic Tension Pneumocephalus
}

Jin-Sung Lee ${ }^{1}$, Sora Ahn', Ki Seong Eom ${ }^{1}$

Departments of ${ }^{l}$ Neurosurgery and ${ }^{2}$ Surgery, Division of Coloproctology, Wonkwang University Hospital, Iksan, Korea

No potential conflict of interest relevant to this article was reported.

\begin{abstract}
The entrapment of intracranial air from the check valve system results in a tension pneumocephalus. It should be distinguished from simple pneumocephalus because they are intracranial space-occupying masses that can threaten life. Communicating hydrocephalus is a serious and frequent complication of post-traumatic head injury. Head injury is one of the most common causes in etiopathogenesis of communicating hydrocephalus. Here, we describe a case of a 65-year-old man who developed communicating hydrocephalus after a post-traumatic tension pneumocephalus. To the best of our knowledge, this is the first reported case of communicating hydrocephalus developed after a post-traumatic tension pneumocephalus. Although the exact pathogenic mechanisms underlying the cascade following trauma remain unclear, communicating hydrocephalus after a tension pneumocephalus could be considered a possible complication.
\end{abstract}

Keywords: Tension pneumocephalus / Hydrocephalus / Head injury / Complication

\section{INTRODUCTION}

Traumatic pneumocephalus is a common complication following traumatic head injury, and is characterized by the abnormal entrapment of air within the intracranial space after injury to the meninges [1,2]. With the exception of cases with leaking cerebrospinal fluid (CSF), pneumocephalus itself can be expected to improve with close follow-up. However, tension pneumocephalus often requires emergency surgery if the volume of intracranial air continually increases due to a check valve system, because of the risk of neurological symptoms and loss of consciousness resulting from increased intracranial pressure [2].

Communicating hydrocephalus is characterized by obstructed CSF flow in the subarachnoid space while CSF flow in the ventricular system is normal, and this can result from not only a con-

\footnotetext{
Correspondence: Ki Seong Eom

Department of Neurosurgery, Wonkwang University Hospital, 895 Muwang-ro, Iksan 54538 , Korea

E-mail: kseom@wonkwang.ac.kr

Received June 2, 2016 / Revised October 18, 2016 / Accepted October 20, 2016
}

genital defect but also acquired causes like infection, hemorrhage, and inflammation [3,4]. Because the enlarged ventricular system continuously compresses the brain, the condition should be diagnosed and treated as early as possible, before irreversible changes occur in the brain [4]. Tension pneumocephalus following traumatic brain injury is a relatively rare disease, and communicating hydrocephalus following surgery for tension pneumocephalus has so far never been reported. Thus, the authors report the case of a 65-year-old patient who developed communicating hydrocephalus after undergoing surgery for tension pneumocephalus.

\section{CASE REPORT}

A 65-year-old male patient came to the hospital with multiple traumatic brain injuries caused by a motorcycle accident. He had a history of diabetes and asthma, for which he had been pharmacologically treated for several years. He also had a history of open surgery to treat peritonitis 3 years before. Upon arrival at the hospital, he was in a lethargic state (Glasgow coma scale; E3V5M5), 
with a loss of direct and indirect light reflexes due to damage to the right optic nerve. Skull X-ray and cranial computed tomography (CT) showed comminuted fractures of the skull and multiple areas around the skull base, a Le Fort type II fracture of the facial bones, multiple comminuted fractures of the nose and around the orbit, and a small amount of pneumocephalus in the anterior recess (Fig. 1). Plastic surgeons decided to delay operating on the facial fractures because of severe edema of the face and orbit, and the patient was treated conservatively at the bedside. On day 8 following the trauma, the patient complained of severe headache and nausea, and CT performed on day 8 revealed a large tension pneumocephalus with a tented configuration and noticeably enlarged shadows of air compressing both sides of the frontal lobe (Fig. 2). As an emergency treatment, a rupture of the dura mater in the area of the right anterior cranial fossa was sutured using a subfrontal approach, and fixation surgery was performed on fragments of the comminuted fractures. Four days later, open reduction and fixation surgery were performed on the multiple facial fractures in the department of plastic surgery. On cranial CT performed 2 weeks postsurgery, pneumocephalus was no longer observed (Fig. 3A). Subsequently, the patient did not have any specific abnormal symptoms and was discharged in a favorable condition. Two months after surgery for tension pneumocephalus, however, the patient returned to the hospital with primary complaints of memory loss, problems in walking, and urinary tract dysfunction. On cranial CT taken at that time, a communicating hydrocephalus was observed with an Evans ratio of 41\% (Fig. 3B). Ventriculoperitoneal shunting was performed, after which the symptoms greatly improved. The skull and abdominal X-rays and cranial CT performed post surgery confirmed that the shunts were well positioned in the ventricles and the abdomen (Fig. 4). The patient again returned to the hospital approximately 4 weeks after the procedure, due to a swelling at the shunt insertion site on the right side of the abdomen. An abdomen CT revealed that the shunt had moved from the intraperitoneal space into the abdominal subcutis, and formed a pseudocyst filled with CSF (Fig. 5). Surgery was per-
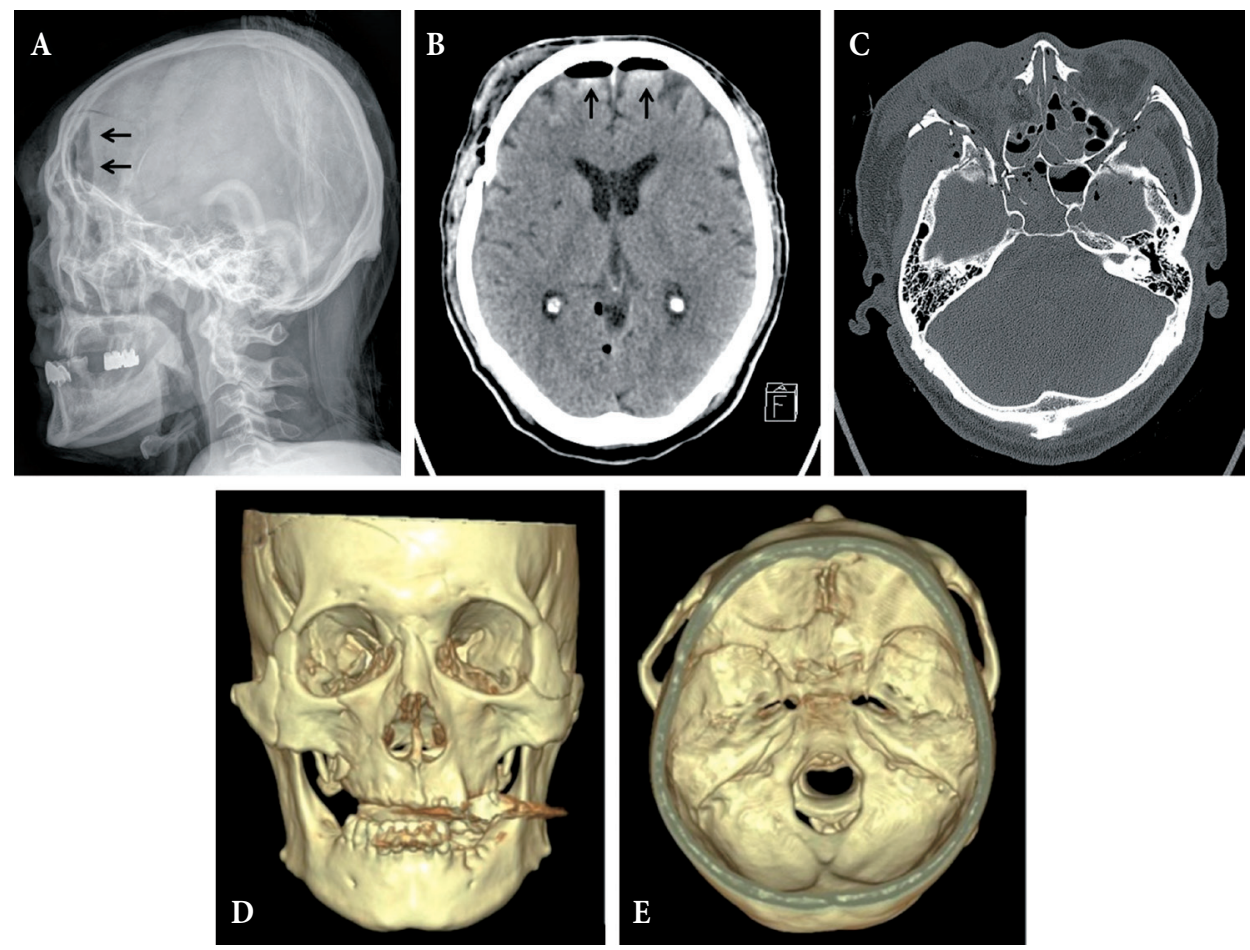

Fig. 1. Skull X-ray (A) and cranial computed tomography (B-E) images showing comminuted fractures on sphenoid and mid facial bones (Le-fort type II fracture), multiple fractures on right frontotemporal bone, nasal bone, right lacrimal bone, and right orbital wall and fissure suggest the right lacrimal gland and optic nerve injuries, and multiple anterior basal skull fractures with small amount of pneumocephalus (black arrows). 

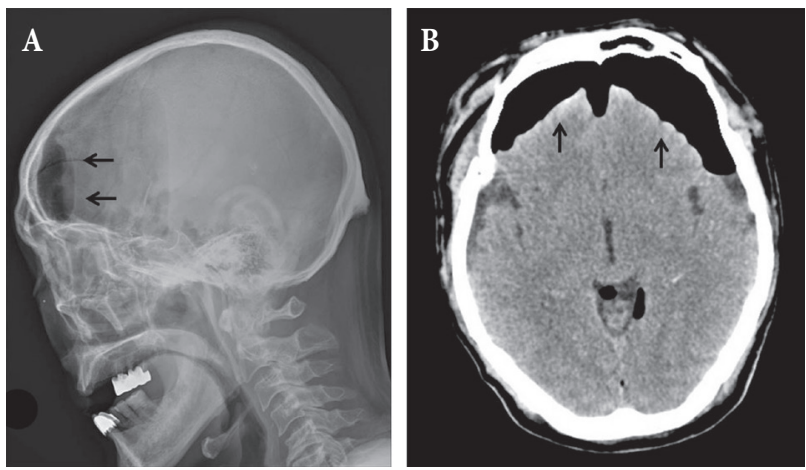

Fig. 2. Skull X-ray (A) and cranial computed tomography (B) images obtained 8 days after admission showing significant increase amount of large pneumocephalus on both frontal areas with tented configuration (black arrows).
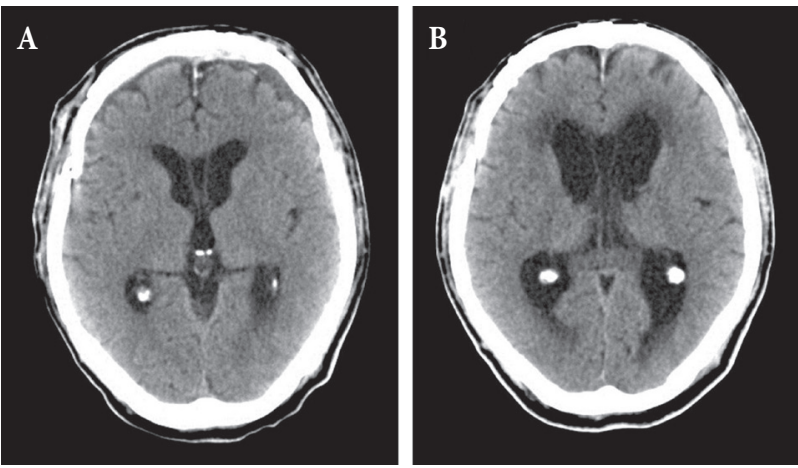

Fig. 3. (A) Cranial computed tomography (CT) image obtained 14 days after operation showing normal size of ventricles without significant pneumocephalus. (B) CT image obtained 14 days after operation showing increased size of all ventricles with periventricular hypolucency suggesting communicating hydrocephalus (Evans ratio $41 \%$ ).
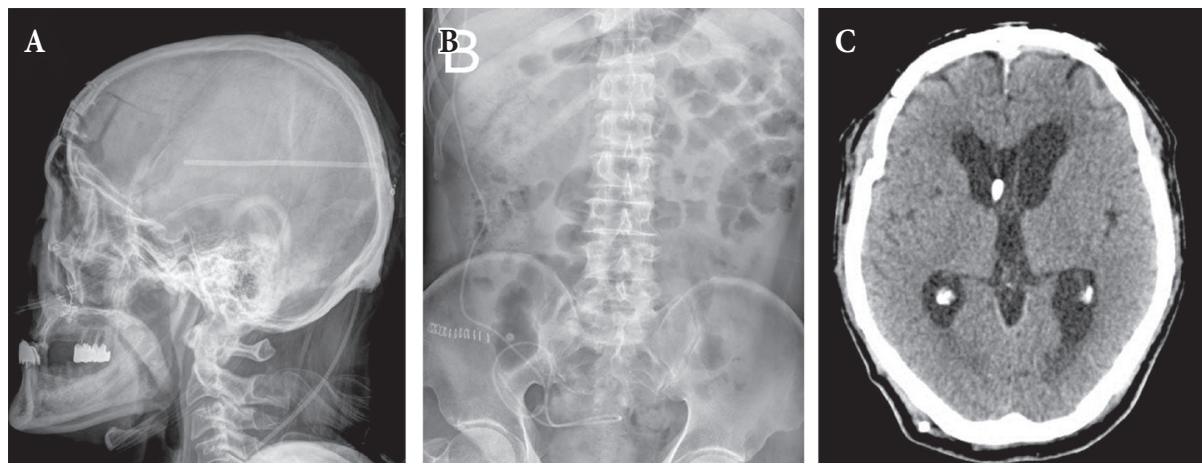

Fig. 4. Skull and abdominal X-rays (A, B) and cranial computed tomography (C) showing well locations of shunt catheter on ventricle and abdominal cavity.
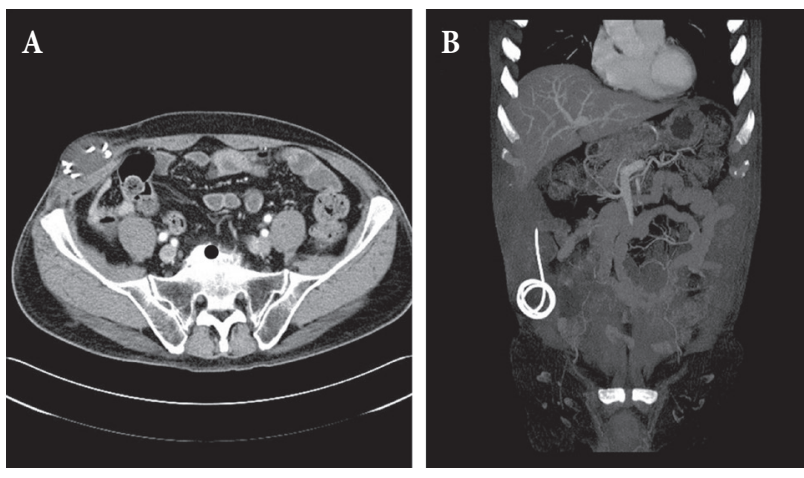

Fig. 5. Axial (A) and coronal (B) abdominal computed tomography showing localized pseudocyst contained distal shunt catheter in right lower abdominal wall.

formed to reinsert the shunt into the intraperitoneal space, and currently ( 3 months after the last procedure), the patient does not have any abnormalities and is in good condition.

\section{DISCUSSION}

Pneumocephalus suggests communication between the intracranial and extracranial spaces, and mostly occurs after trauma. However, it may also occur, albeit rarely, following sinus surgery, brain surgery, or lumbar puncture, or due to a secondary bone defect such as infection caused by gas-forming bacteria or osteomyelitis [1,2]. Regarding the mechanism, one hypothesis suggests that air enters the skull cavity to compensate for a decrease in intracranial pressure due to a CSF leak, while another explanation is that air enters a defect in the skull and dura mater when sinus pressure increases, such as during a sneeze [5,6]. In most cases, pneumocephalus involves low pressure and resolves spontaneously within days. However, if air continually accumulates due to the presence of a check valve system in the damaged area, tension 
pneumocephalus can occur, although rarely, with a serious mass effect $[1,5]$. It is an acute condition that can sometimes cause serious neurological complications, and therefore an accurate diagnosis and prompt treatment are required [2]. It has been reported that tension pneumocephalus in the subdural space with a volume of air over $65 \mathrm{~cm}^{3}$, as seen in the patient reported in this case study, occurs in under $8 \%$ of patients with basilar skull fractures including fracture of the sinuses [6,7]. On CT images, tension pneumocephalus in the subdural space typically shows a tented configuration in which trapped air located in the front compresses both frontal lobes (Fig. 2) [6].

In general, hydrocephalus is characterized by an enlarged ventricle resulting from dysfunctional CSF flow, either because the CSF pressure is excessively high or CSF pathways are obstructed $[4,8]$. Depending on the mechanism, hydrocephalus is classified either as non-obstructive hydrocephalus caused by CSF overproduction due a choroid plexus tumor for instance, or as obstructive hydrocephalus caused by dysfunctional CSF flow. Most cases of hydrocephalus are obstructive hydrocephalus, which is further classified depending on the location of the obstruction, as either a non-communicating hydrocephalus in which intraventricular obstruction occurs or as communicating hydrocephalus in which obstruction occurs in an area below the fourth ventricle. Communicating hydrocephalus occurs mainly due to subarachnoid hemorrhage or meningitis, whereas non-communicating hydrocephalus occurs due to an obstruction in the ventricular system caused by intraventricular tumor, hematoma, or brain herniation [8]. Hydrocephalus following traumatic brain injury was first reported in 1914 by Dandy. The incidence rate varies widely with a range of $8 \%-72 \%$, and it has been reported that $1 \%-4 \%$ of cases require shunting [8-10]. For many years, shunting has been a universal surgical procedure for hydrocephalus [3], but complications can occur such as a blockage in the shunt, change in the location of the shunt, a mechanical defect like a problematic valve, slit ventricle syndrome, infection, intestinal perforation, subdural hematoma, or the formation of a pseudocyst around the peritoneum $[3,8]$. Our patient developed a pseudocyst, as the shunt moved to the abdominal subcutis due to intestinal adhesions following past surgery for peritonitis. He underwent a second procedure, but re- peat surgery performed in the presence of complications can be a primary cause of increased morbidity and mortality and care should be taken [3].

In our patient, communicating hydrocephalus occurred following tension pneumocephalus. The presence of a significant hematoma in the subarachnoid space was not confirmed, but we suspect hemorrhage resulted from the basilar skull fracture. Dryness or damage over a relatively large area of the subarachnoid space and arachnoid villi due to tension pneumocephalus would have contributed to dysfunctional CSF flow. Until now, communicating hydrocephalus following traumatic tension pneumocephalus had not been reported and is a very rare complication, but tension pneumocephalus can be acquired after communicating hydrocephalus. Therefore, we suggest that patients should be continuously followed up and examined neurologically following surgery for tension pneumocephalus.

\section{REFERENCES}

1. Dabdoub CB, Salas G, Silveira Edo N, Dabdoub CF. Review of the management of pneumocephalus. Surg Neurol Int 2015;6:155.

2. Sweni S, Senthilkumaran S, Balamurugan N, Thirumalaikolundusubramanian P. Tension pneumocephalus: a case report with review of literature. Emerg Radiol 2013;20:573-8.

3. Reddy GK, Bollam P, Caldito G. Long-term outcomes of ventriculoperitoneal shunt surgery in patients with hydrocephalus. World Neurosurg 2014;81:404-10.

4. Tian HL, Xu T, Hu J, Cui YH, Chen H, Zhou LF. Risk factors related to hydrocephalus after traumatic subarachnoid hemorrhage. Surg Neurol 2008;69:241-6.

5. Jacobs JB, Persky MS. Traumatic pneumocephalus. Laryngoscope 1980;90:515-21.

6. Bae WK. Imaging of head injury. In: Hwang SC, editor. Neurotrauma. 2nd ed. Seoul: Koonja; 2014. p. 70-1.

7. Monajati A, Cotanch WW. Subdural tension pneumocephalus following surgery. J Comput Assist Tomogr 1982;6:902-6.

8. Hyun DK. Neurosurgical sequale. In: Hwang SC, editor. Neurotrauma. 2nd ed. Seoul: Koonja; 2014. p. 351-2.

9. Guyot LL, Michael DB. Post-traumatic hydrocephalus. Neurol Res 2000;22:25-8.

10. Gudeman SK, Kishore PR, Becker DP, Lipper MH, Girevendulis AK, Jeffries BF, et al. Computed tomography in the evaluation of incidence and significance of post-traumatic hydrocephalus. Radiology 1981;141:397-402. 